\section{Controlling heart rhythm reduces adverse outcomes in atrial fibrillation}

Around $2 \%$ of the European population suffer from atrial fibrillation, a heart condition characterised by a change in heart rhythm that increases the risk of serious health complications. Currently, therapies to directly address the arrhythmia are not used for all patients. The Atrial in brillation Network 4 trial which set out to test whether systematic early control therapy prevents adverse outcomes in patients with atrial fibrillation (AF) compared to usual care relying on restricted symptom-driven use of rhythm control therapy. The main trial results and careful subanalyses demonstrate that early, systematic use of rhythm control can reduce AF-related complications by one fifth when added to the best currently available treatment. Implementation of early rhythm control therapy in patients with atrial fibrillation can help to reduce the devastating consequences of the arhythmia, such as cardiovascular death, stroke, and heart failure.

\section{trial fibrillation (AF) is the most}

common form of cardiac
arrhythmia and disrupts th ormal rhythm of the heart. AF causes an egular (often abnormally fast) heartbeat, dizziness, and shortness of breath. Some people feel their heart tuttering or experience any symptoms.

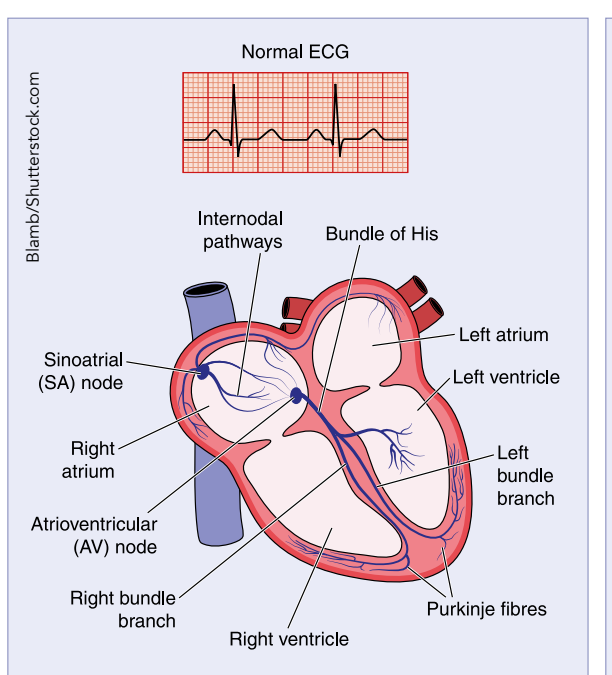

Although AF is often not recognised, so-called asymptomatic AF, even thes cant cause cardiovascular death, stroke, or

Normally, the muscles of the heart

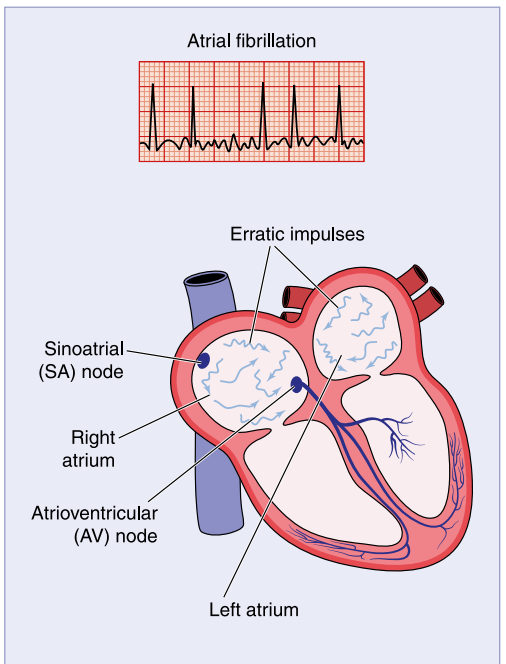

upper chambers of the heart, called the atria, are activated randomly. They stop contracting and heventicles, the main heart chambers, contract inegularly. This relax between contractions, reducing its efficiency and performance. countries is rising, and it is estimated that the incidence of AF will have rise $250 \%$ by 2050 .

Treatment involves ruling out other causes of $A F$, such as an overactive thyroid. In addition, strokes related to thimners (anticoagulants). In addition, medications ('rate control therapy') and cardion and herapy of concomitant compascular conditions are important with AF.

In addition, antiarryhthmic drugs, cardioversion, and atrial fibrillation ablation are available to restore and maintain the normal heart rhythm in patients with atrial fibrillation. These treatments are called thythm control therapy. Twenty years ago, several controlled trials did not find a clinical benefit of rhythm control therapy. some of the initial trials suggested that rhythm control therapy was associated Therefore, most patients are currently not offered rhythm control therapy. Rhythm control is restricted to symptomatic patents. Prior to the EAST-AFNET 4 trial, there were only small studies in special
The number of patients who are Af can be prevented by taking blood slowing a fast heart rate during AF using with a higher number of hospitalisations.
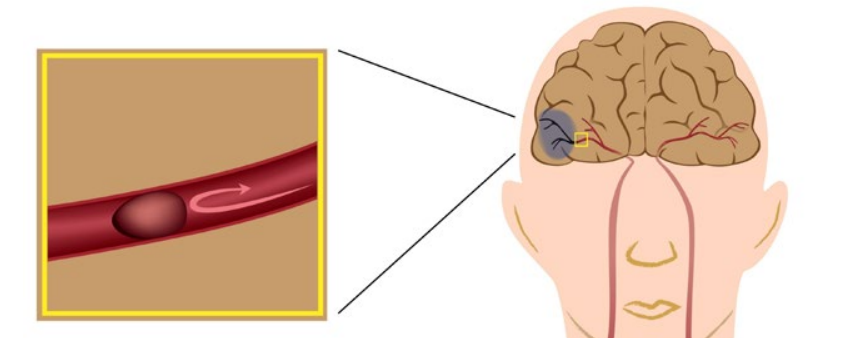

In atrial fibrillation, the
upperc chambers upper chambers of
the heart (atria) do not pumpe efficiently. This
results in an increased results in an increased
risk of blood clots

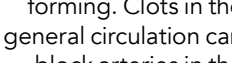

3. Clot blocks an artery in the brain
causing stroke

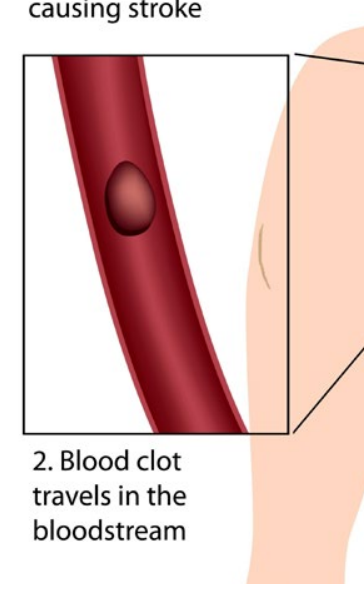

with AF and heart failure, in whom a lower rate of cardiovascular events was found.

Despite these treatments, people with Af remain at higher risk of heart and

One of the risks associated with AF is an increased chance of experiencing a stroke. individuals with $\mathrm{A}$ are five circulatory problems. This risk is also increased during the first year after times more likely to have a stroke atrial fibrillation' A Aden called 'early heart failure, stroke, and death still occur

Atrial fibrillation is the most common form of cardiac arrhythmia and disrupts the normal rhythm of the heart.

than other people. Therefore, many

This is currently mainly achieved by

using medications to thin the blood

to prevent blood clots forming which

could cause a stroke. They may also be prescribed drugs to lower their

heart rate.

suggesting that the current approach to treating AF requires further optimisation become more readily available.

Despite the findings in the old 'rate versus rhythm' control trials, the investigators of the Early treatment of Atrial fibrillation for
STroke prevention (EAST-AFNET 4) tria and others were aware of mechanistic better for the heart and for patients wis $A F$, than remaining in atrial fibrillation. These considerations led to the design and conduct of the EAST-AFNET 4 trial.

\section{EAST-AFNET 4}

Previous studies suggest that rhythm control therapy is only recommended to alleviate AF-related symptoms, although it has the potential to eliminate the arrhythmia itself. The results of these studes also indirectly showed that rhythm confrol herapy, used in conjunction with alcoagulants, helped to prevent blood (normal heart rhythm).

The EAST-AFNET 4 trial aimed to test whether restoring a normal heart rhythm soon after diagnosis of $A F$ can reduce the residual risk of health complications.

To meet the inclusion criteria for the study patients had to have been diagnosed with AF less than a year before enrolment, as well as having a cardiovascular conditions such as a previous stroke, diabetes,

The participants were then randomly assigned to one of two groups: the first received systematic, early rhythm contro and the second group received usual care. Early rhythm control included either treatment with antiarrhythmic drugs or atrial fibrillation ablation (a procedure that carefully removes damaged heart tissue

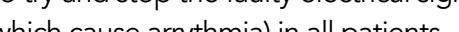
directly after randomisation. The cho of treatment was decided by the team looking after the patient. Treatment was adjusted based on detection of recurren AF episodes: $95 \%$ of the patients randomised to early rhythm contro received rhythm control therapy.

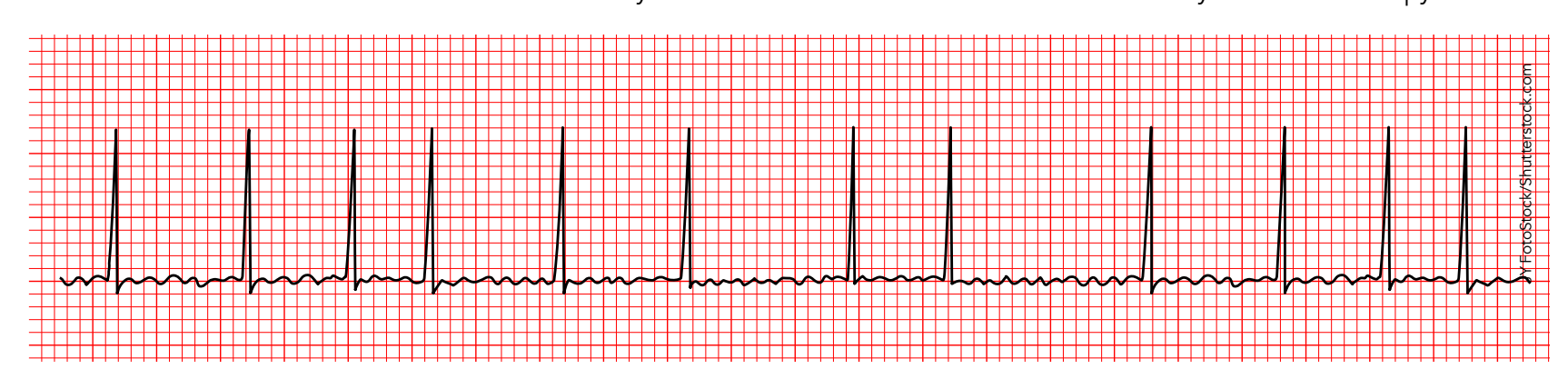




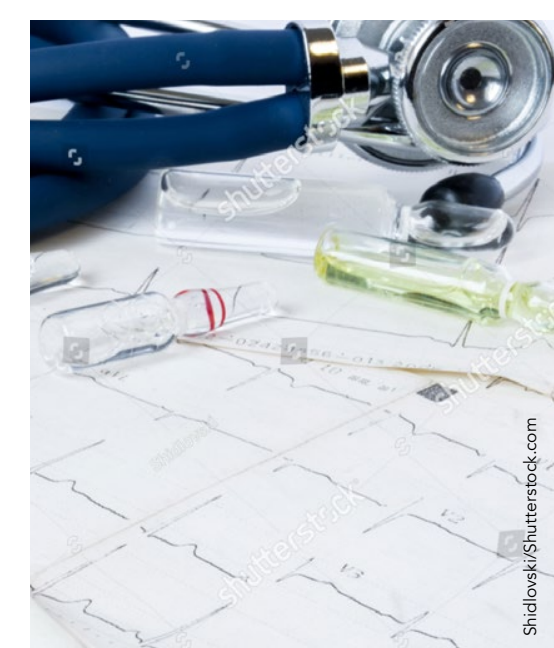

Current guidelines recommend hrythm
control therapy only for Af patients who have

The usual care group received The usual care group received
anticoagulation, rate control, and therapy of concomitant cardiovascular patients randomised to early rhythm control. Rhythm control treatment was only used to improve atrial fibrillationrelated symptoms, aligned with current ecommendations. Overall, only one in seven patients randomised to usual care received rhythm control therapy.

Outcomes included cardiovascular complications, such as death, stroke, and hospitalisation for heart failure or

The researchers recruited 2,789 different centres inerent centres and patients were followed up for an verage of 5.1 years.

The results of the clinical trial showed that treatments which returned the heart to a normal rhythm reduced the risk of cardiovascular complications, including stroke and cardiovascular death, by around $20 \%$. This improvement was observed in a wide range of patients with $A F$, including those with no symptoms, those with and without heart heart rhythm at randomisation.

The researchers also explored possible easons for the clinical benefits seen

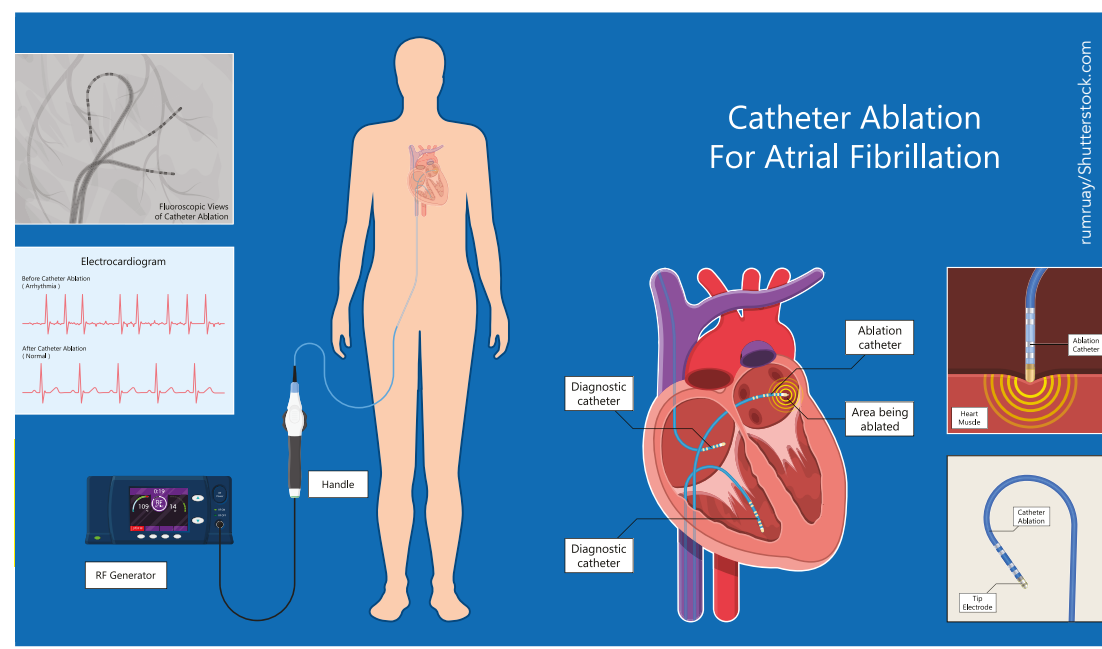

The EAST-AFNET 4 trial provides evidence that starting rhythm control therapy soon after diagnosis

with early rhythm control. They found that there were no differences in other coagulation medications, that cous an explain the difference between the control and the early therapy group.

Finally, both treatment approaches were equally safe for patients. Although there were fewer deaths in patients treated with early rhythm control therapy, compared to the usual control group, this was not statistically significant. Overall, early rhythm control therapy

outcome of death or hospitalisation for heart failure.

There was also no difference in outcom when symptomatic and asymptomatic (or silent) patient groups were compared in a further sub-analysis, suggesting is beneficial for all patients with newly diagnosed AF, regardless of how their condition presents itself.

FURTHER SUB-STUDIES

AND ANALYSES

Currently, clinical guidelines recommen wherapy only for patients who have persistent arly rhythm control is an opportunity wher This means that, despite the positive effect that hythm control therapy available therapies.

require patients to spend longer in hospital and was associated with a lower risk of adverse cardiovascular outcomes than usual care in patien with recently diagnosed AF and diovascular conditions.

A sub-analysis comprising of 798 patients from the EAST-AFNET 4 trial also supports this finding in patients with heart failure, suggesting that the presence of other health condition is not a contraindication to early conth control therapy. Early rhyth can have for AF patients, it is only used to treat around one in seven patients with AF in Europe.

The EAST-AFNET 4 trial provides evidence that starting rhythm contro therapy soon after diagnosis can improve outcomes in patients with to patients who are symptomatic or asymptomatic and applies regardless failure The findings will info as heart diagnosed atrial fibrillation.

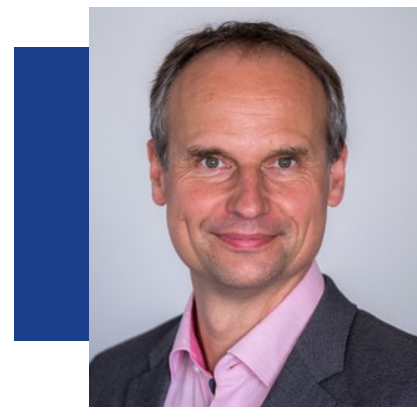

\section{Behind the Research}

\section{Professor Paulus Kirchhof}

E: p.kirchhof@uke.de E: info@af-net.eu T: +4940741052438

W: www.kompetenznetz-vorhofflimmern.de that early, systematic rhythm control

Research Objectives

The EAST-AFNET 4 trial set out to explore the effects of early control therapy on patients with atrial fibrillation.

\section{Detail}

Address

University Medical Center Hamburg-Eppendor University Heart and Vascular Center Department for Cardiology

Atrial Fibrillation NETwork

Münster, Germany

W: www.kompetenznetz-vorhofflimmern.de

Paulus Kirchhof is Director of the Department of Cardiology at the University Heart and Vascular Center UKE Hamburg, and holds a part-time position as Professor of Cardiovascular Medicine at the University of Birmingham, UK. He studied medicine in Heidelberg, Lille, Georgetown and Münster. After graduating, Professor Kirchhof learned and practiced cardiology at the University Hospital Munster. He worked in Bimingham from 2011, moving to Hamburg in 2020. He researches translational mechanism and management of cardiovascular diseases, especially any AF. This finding can be extrapolated fallure. The findings will inform clinica
Funding

EHRA, Deutsche Herzstiftung (DHS), Abbott Laboratories, Sanofi

C 5 study centres, EHRA, AFNET (sponsor of the study)

early treatment of atrial fibrillation for stroke prevention tria

\section{References}

Metzner, A, Suling, A, Brandes, A, et al, (2021) Anticoagulation, therapy of concomitant conditions, and early rhythm control therapy: a detailed analysis of treatment patterns in the EAST-AFNET 4 trial. Europace, doi:10.1093/ europace/euab200

Rillig, A, Magnussen, C, Ozga, AK, et al, (2021) Early rhythm control therapy in patients with atrial fibrillation and

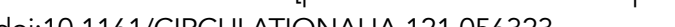
doi:10.1161/CIRCULATIONAHA.121.056323

Willems, S, Borof, K, Brandes, A, et al, (2021) Systematic, early rhythm control strategy for atrial fibrillation in patients with or Journal, doi-10.1093/enheari/ehab593

Kirchhof, P, Camm, AJ, Goette, A, et al, for the EAST-AFNET 4 trial investigators (2020) Early rhythm control therapy in patients with atrial fibrillation. New Engl J Med, doi:10.1056 NEJMoa2019422

\section{Personal Response}

Why is early rhythm control therapy not currently recommended by more clinical guidelines?

II The trial results were reported at the same time as the 2020 European Society of Cardiology atrial fibrillation the findings into new guidelines. I am confident that international guideline committees will look into updating their guidelines at this point in time.

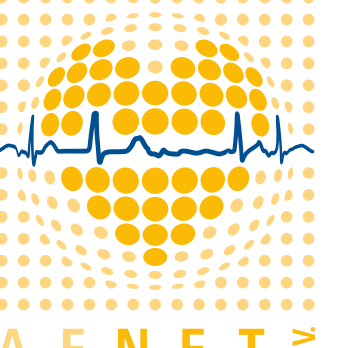

Atrial Fibrillation NETWORK 